\title{
Deep Brain Stimulation for Epilepsy
}

\author{
Casey H. Halpern,* Uzma Samadani,* Brian Litt, ${ }^{\dagger}$ Jurg L. Jaggi,* and Gordon H. Baltuch* \\ *Department of Neurosurgery and ${ }^{\dagger}$ Department of Neurology, Center for Functional and Restorative Neurosurgery, University of \\ Pennsylvania Medical Center, Philadelphia, Pennsylvania 19104
}

\begin{abstract}
Summary: Many patients who suffer from medically refractory epilepsy are not candidates for resective brain surgery. Success of deep brain stimulation (DBS) in relieving a significant number of symptoms of various movement disorders paved the way for investigations into this modality for epilepsy. Open-label and small blinded trials have provided promising evidence for the use of DBS in refractory seizures, and the first randomized control trial of DBS of the anterior thalamic nucleus is currently underway.
\end{abstract}

There are multiple potential targets, because many neural regions have been implicated in seizure propagation. Thus, it is difficult as yet to make any definitive judgments about the efficacy of DBS for seizure control. Future study is necessary to identify a patient population for whom this technique would be indicated, the most efficacious target, and optimal stimulation parameters. Key Words: Deep brain stimulation, epilepsy, thalamus, seizure, closed-loop systems.

\section{INTRODUCTION}

Despite maximal antiepileptic drug (AED) therapy, more than $30 \%$ of patients with epilepsy will suffer from persistent seizures. ${ }^{1-3}$ Resective brain surgery is generally indicated for refractory partial seizures and can provide at least a $90 \%$ reduction in seizure frequency ${ }^{4}$; however, permanent neurologic deficits or death occurs in nearly $4 \%$ of cases. ${ }^{4}$ Furthermore, up to $40 \%$ of patients are not candidates for surgical resection. ${ }^{5}$ Vagus nerve stimulation (VNS) as an adjunct to medical therapy is a reasonable option for these patients and is associated with up to a $50 \%$ reduction in seizure frequency. ${ }^{6}$ Nevertheless, most of these patients will not be seizurefree. Thus, due to the remarkable success of deep brain stimulation (DBS) for movement disorders, ${ }^{7,8}$ combined with its advantages of titratability, reversibility, and low risk of complications, ${ }^{9,10}$ there has been an explosion of research into implantable deep brain devices for treating refractory epilepsy. ${ }^{11,12}$

The mechanism by which DBS may diminish seizures remains largely unknown. The clinical effect of highfrequency DBS, however, appears to mimic precursor ablative procedures. ${ }^{13}$ Thus, stimulation-induced disruption of unopposed network activity is one hypothesis that

Address correspondence and reprint requests to: Gordon Baltuch, M.D., Ph.D., F.R.C.S.C., Department of Neurosurgery, 3 Silverstein, Hospital of the University of Pennsylvania, 3400 Spruce Street, Philadelphia, PA 19104. E-mail: gordon.baltuch@uphs.upenn.edu. appears to be consistent with available data. ${ }^{14,15}$ Furthermore, DBS at high frequencies may block cortical epileptiform activity. ${ }^{16-18}$ Indeed, the anterior nucleus of the thalamus (ANT) has been lesioned stereotactically, resulting in improved seizure control in humans. ${ }^{19}$ DBS of various neural targets has been investigated, including the thalamus, the subthalamic nucleus (STN), cerebellum, hippocampus, caudate nucleus, and mammillary nuclei. $^{20-24}$ Here we will review evidence from animal studies and rare pilot studies in humans of ANT DBS, which is currently being evaluated as a potential target in an ongoing double-blind randomized trial in humans (the SANTE trial: Stimulation of the Anterior Nucleus of the Thalamus for Epilepsy). ${ }^{25}$

\section{DEEP BRAIN STIMULATION OF THE ANTERIOR NUCLEUS OF THE THALAMUS}

\section{Rationale}

In 1937, James W. Papez ${ }^{26}$ described a circuit linking hippocampal output via the fornix and mammillary nuclei to the ANT. Projections from the ANT travel to the cingulum bundle and then around the wall of the lateral ventricle to the parahippocampal cortex, completing the circuit by returning to the hippocampus. Radiologic alterations have been noted in structures within the classic circuit of Papez in mesial temporal sclerosis, as well as other forms of epilepsy. ${ }^{27}$ Thus, it is conceivable that DBS of structures within this circuit may result in direct 
anterograde cortical stimulation. The ANT is an appealing target because of its relatively small size and projections to limbic structures, ultimately affecting wide regions of neocortex. Further, it is not as deep or close to basal vascular structures as are the mammillary nuclei. These anatomic connections and precursor ablative techniques have motivated investigation of ANT DBS for epilepsy.

\section{Animal studies}

Seizure suppression with electrical stimulation of deep brain structures has shown promise in animal models. Observations of enhanced glucose metabolism in the ANT after administration of both pentylenetetrazole and ethosuximide to guinea pigs supports the role of the ANT in propagating seizure activity. ${ }^{28}$ Bilateral electrolytic lesions of the tracts connecting the mammillary bodies to the ANT result in essentially complete protection from pentylenetetrazol-induced seizure activity. ${ }^{29}$ Recently, high-frequency DBS $(100 \mathrm{~Hz})$ of the ANT has been shown to increase the seizure threshold in a pentylenetetrazole-induced seizure model. ${ }^{30}$ On the other hand, lowfrequency $(8 \mathrm{~Hz})$ DBS was proconvulsant, as noted in earlier studies. ${ }^{18,31}$ Bilateral ANT DBS may also prolong the latency of onset of status epilepticus after administration of pilocarpine. ${ }^{32}$ Nevertheless, efficacy of ANT DBS for epilepsy in animal models is still debated, given its lack of effect reported from numerous studies, as well as an exacerbation of seizure frequency by chronic ANT DBS. ${ }^{32,33}$

\section{Potential indications in humans}

Currently there are no widespread indications for DBS in epilepsy, because it is still considered an experimental therapy. For inclusion in the SANTE trial, patients must suffer from partial onset seizures with or without secondary generalization associated with frequent falls, injuries, and impaired quality of life. ${ }^{34-37}$ Seizures should be refractory to $12-18$ months of at least two therapeutically dosed antiepileptic agents. In the future, however, a failure to respond to surgical resection or vagal nerve stimulation may also be indications for DBS. Scalp video-EEG monitoring is necessary to characterize seizures and demonstrate bilateral or nonlocalizing findings consistent with preoperative imaging. The subject, family, or legal guardian must be capable of recognizing seizures and maintaining a seizure diary.

\section{ANT targeting}

At our center, general endotracheal anesthesia or local anesthesia is administered before placement of the stereotactic frame. ${ }^{38}$ Frame tilt should be parallel to the lateral canthal-external auditory meatal line, which is itself approximately parallel to the anterior commissureposterior commissure (AC-PC) line. After attachment of the magnetic resonance imaging (MRI) localizer, an MRI is obtained with both fast spin echo inversion recovery and standard T2 images. ${ }^{34-37}$ Computed tomography (CT) may be a reasonable alternative to MRI for stereotactic localization of the ANT. ${ }^{34}$

Target sites are selected from MRI by using 1-mm thick-axial, coronal, and sagittal spoiled gradient echo pulse sequences. Indirect localization of the ANT is performed with reference to a standard stereotactic atlas ${ }^{40}$ by identifying the AC-PC line on the sagittal image. AC and $\mathrm{PC}$ coordinates relative to the center of the frame are obtained to calculate the midcommissural point. The ANT is $5 \mathrm{~mm}$ lateral and $12 \mathrm{~mm}$ superior to the midcommissural point. ${ }^{40}$ Direct visualization of the ANT is also possible. We download the imaging data to a surgical navigation system (Medtronic Stealth navigation; Medtronic, Minneapolis, MN; Stryker Leibinger, Kalamazoo, MI), to perform simulation of the planned trajectory from the entry point to the ANT, to confirm avoidance of sulcal vessels (FIG. 1). Because of the location of the ANT, the lateral ventricle is invariably traversed.

\section{Surgical procedure}

The patient is placed in a supine or semisitting position. ${ }^{34-39}$ After Mayfield fixation, sterile preparation of the unshaven head, and local infiltration with $1 \%$ lidocaine 1:100,000 epinephrine, an incision is made overlying the coronal suture. A burr hole is placed, and the dura and pia are sharply incised and cauterized. A guide cannula is inserted and advanced deep into the brain to a point $10 \mathrm{~mm}$ from the desired target under direct fluoroscopic and Leksell frame guidance. Under local anesthesia, a monopolar single-unit recording electrode (Advanced Research Systems, Atlanta, GA, or FHC, Bowdoinham, ME) can be introduced to confirm anatomic depth for entry into thalamic tissue after traversing the lateral ventricle. ${ }^{35}$ No units are recorded while traversing the lateral ventricle. The electrode tip is advanced until recordings are first heard (ANT superficial surface), then until units cease (intralaminar region) and then recommence (dorsomedian nucleus of the thalamus). Extracellular action potentials are amplified with a GS3000 (Axon Instruments, Sunnyvale, CA) or Leadpoint (Medtronic) amplifier and simultaneously recorded using standard recording techniques $(300-10,000 \mathrm{~Hz})$, together with a descriptive voice channel. ANT neurons are identified based on 1) regional characteristics, 2) a firing rate previously described for human recordings,${ }^{35}$ and 3 ) a characteristic burst firing pattern.

After removing the single-unit recording electrode, a stimulation lead (Radionics stimulation/lesioning probe; Integra Radionics, Burlington, MA) is introduced to elicit the driving response suggestive of ANT placement with a stimulation frequency of 5 to $10 \mathrm{cycles} / \mathrm{s}$, pulse width at 90-330 $\mu$ s, pulse amplitude at 4-5 V, and total pulse durations between 3 and $10 \mathrm{~s}$ (FIG. 2). 


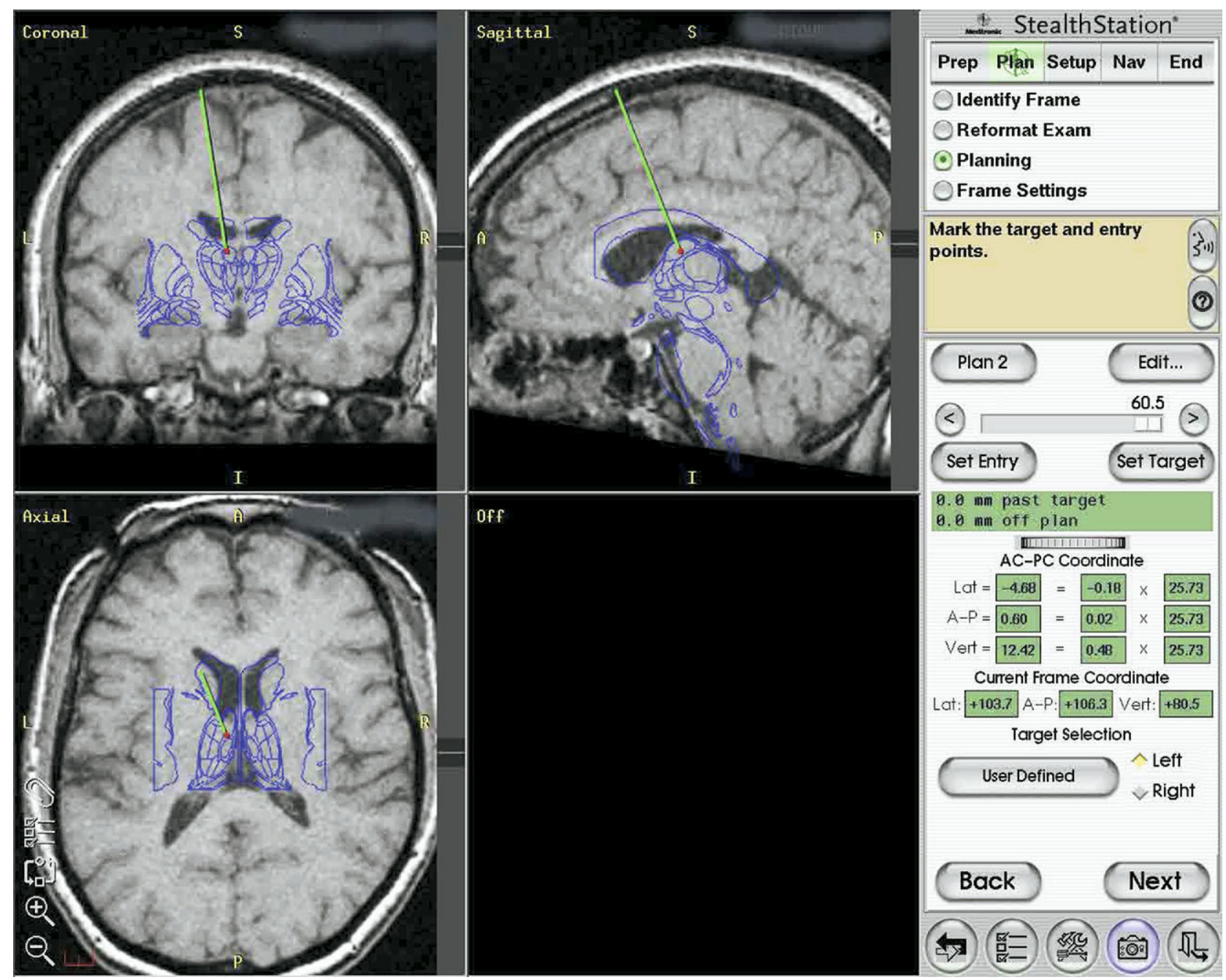

FIG. 1. Diagram demonstrating simulation of the planned trajectory to the anterior nucleus of the thalamus for deep brain stimulation using a surgical navigation system (Medtronic Stealth navigation; Medtronic, Minneapolis, MN) with a superimposed standard stereotactic atlas.

ANT DBS has been associated with recruiting rhythms on cortical EEG in patients with the most pronounced seizure frequency reduction. ${ }^{36}$ These changes in EEG signal morphology can, however, be elicited from several other thalamic nuclei. ${ }^{31}$ Furthermore, a recent report indicated that these recruiting rhythms induced by lowfrequency ANT DBS may reflect mixed activation of both specific and nonspecific thalamocortical pathways, and thus not serve as a physiologic verification of optimal localization. ${ }^{41}$

Once this lead has been removed, the DBS lead is advanced to the ANT, ensuring that all contacts of the lead are within thalamic parenchyma, and the cannula and lead stylet are withdrawn under fluoroscopic guidance. The stimulation leads we use are Medtronic 3387 DBS depth electrodes with 4 platinum-iridium stimulation contacts $1.5 \mathrm{~mm}$ wide, with $1.5 \mathrm{~mm}$ edge-to-edge separation because the ANT is relatively larger than other DBS targets. Another fluoroscopic image is acquired to show that the electrode is secure. The lead is secured to a burr-hole cap, and the skin incision is closed. The same sequence of steps is performed to place the contralateral electrode. The stereotactic frame is removed.

We elect to place the internal pulse generator (IPG) at the same setting, but it can be placed at a later date. The head, neck, and infraclavicular regions are sterilized in preparation for placement of the IPG (Medtronic model Itrel II, Soletra, or Kinetra) in a subclavicular pocket bilaterally. The scalp incision is reopened for connection of the lead to an extension wire (Medtronic 7495 lead extension), which is tunneled subcutaneously to the IPG or IPGs.

\section{Postoperative management}

The site of placement of the DBS leads is confirmed postoperatively by MRI or CT after reversal and recovery from anesthesia (FIG. 3). ${ }^{34-39}$ Patients are discharged to home 2 days postoperatively, but multiple 


\section{Stimulation L Anterior Nucleus: Driving Response} $6 \mathrm{~V}, 10 \mathrm{cps}, 330$ usec pulse width

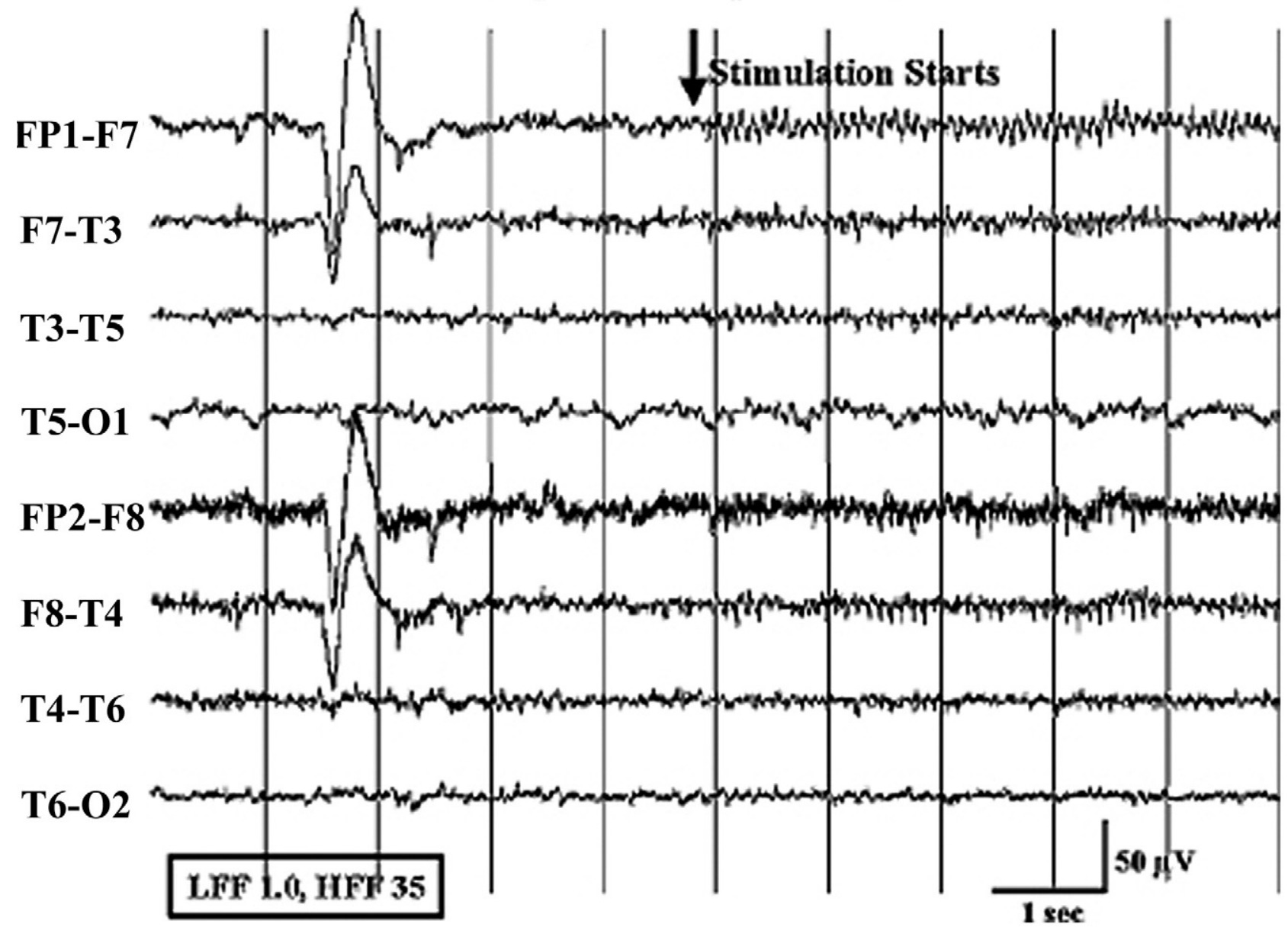

FIG. 2. Scalp EEG driving response to stimulation of the left-sided anterior nucleus of the thalamus at a frequency of $5-10 \mathrm{~Hz}$, pulse width at $90-330 \mu \mathrm{s}$, pulse amplitude at $4-5 \mathrm{~V}$, and total pulse duration between 3 and $10 \mathrm{~s}$. (Adapted from Kerrigan et al. ${ }^{35}$ )

outpatient visits are usually necessary for optimization of stimulation parameters. Stimulators are turned on at $\sim 10$ days postoperatively, and initial stimulation parameters are set at a frequency of $90-130 \mathrm{~Hz}$, pulse width at 60-90 $\mu$ s, and pulse amplitude at 4-5 V, and adjusted during subsequent follow-up to maximize clinical benefit based on each individual response and minimize side effects, such as nystagmus, lethargy, and anorexia. ${ }^{39}$

The benefits of bipolar versus monopolar stimulation remain to be determined as do cycling versus continuous stimulation. A recent study of bilateral ANT DBS in four patients with refractory epilepsy found no significant difference in seizure frequency between cycling and continuous stimulation period using within-group comparisons. ${ }^{34}$ One proposed method of cycling or intermittent stimulation using an open-loop device (discussed below) is through a duty cycle of $1 \mathrm{~min}$ on and $5 \mathrm{~min}$ off. ${ }^{11}$ Intermittent stimulation may increase the battery life of the IPG, is theoretically safer for neural tissue than chronic continuous stimulation, and has been successful in clinical trials for VNS for epilepsy. ${ }^{36}$

\section{Clinical outcome}

We are limited at this time to small sample pilot studies evaluating ANT DBS as a treatment for pharmacoresistant seizures. Despite significant individual variation in outcome, bilateral high-frequency ANT DBS appears overall to be safe, well-tolerated, and effective in some subjects with inoperable refractory epilepsy. Table 1 summarizes the results of six pilot studies of ANT DBS.

Upton et al. ${ }^{42}$ reported significant reduction in seizure frequency in four of six patients with intractable epilepsy, one of whom was completely seizure-free at follow-up. This may be due to microthalamotomy or lesioning of the ANT. ${ }^{43}$ Electrode implantation appears to decrease seizure frequency itself, and activation of the IPG and multiple subsequent adjustments to stimulatory parameters or contacts may not be linked to any further 

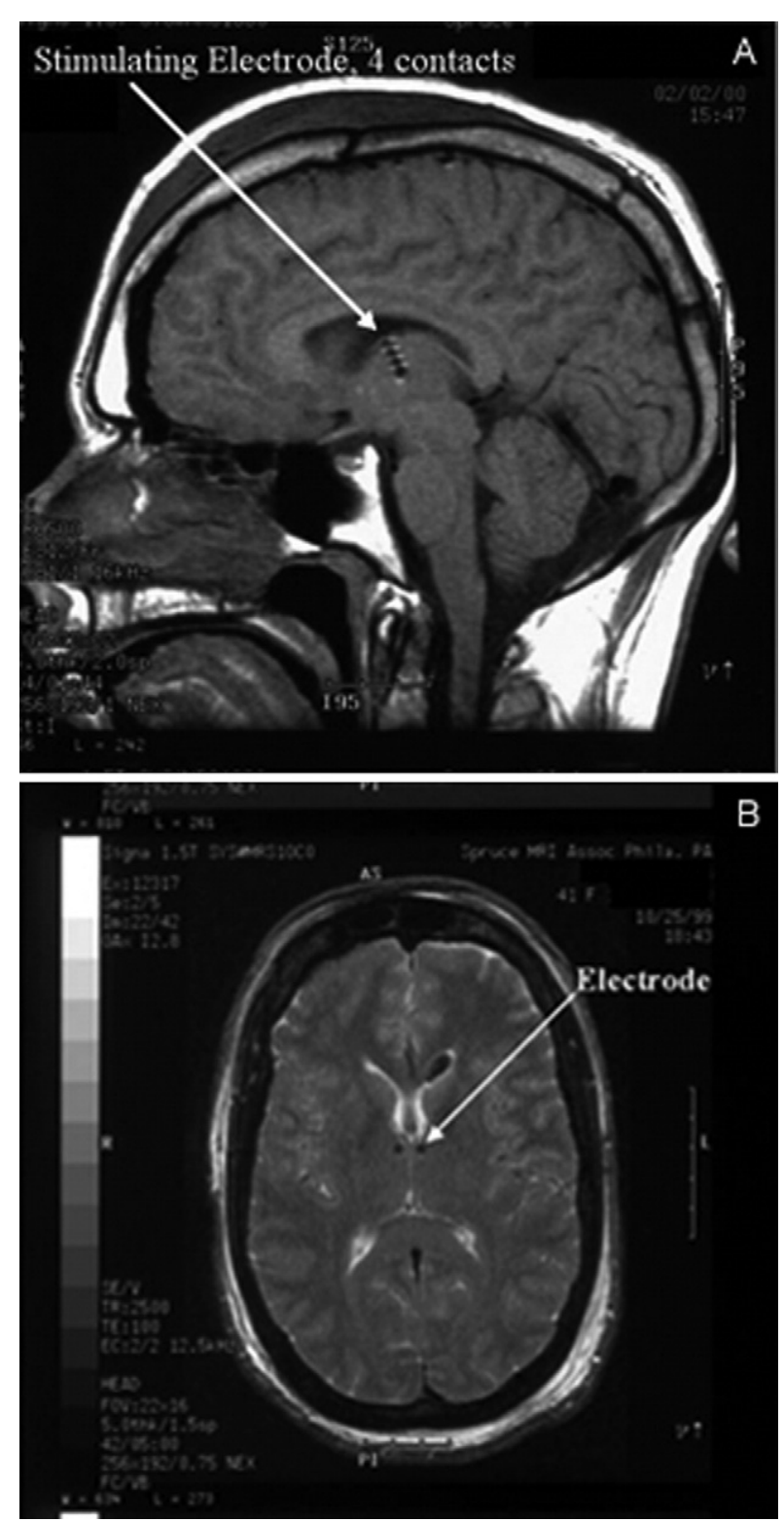

FIG. 3. A T1-weighted sagittal (A) and T2-weighted axial (B) magnetic resonance image demonstrating lead placement for deep brain stimulation of the anterior nucleus of the thalamus. (Adapted from Kerrigan et al. ${ }^{35}$ )

benefits in seizure control. ${ }^{34,39}$ In one patient, however, the IPGs were turned off after 3 years of DBS with maintenance of improved seizure control for more than 1 year. Thus, it is difficult to distinguish the relative effects of electrode insertion and stimulation on the reduction in seizure frequency.

The effect of microthalamotomy on seizure propagation, however, continues to be debated, and a recent study was unable to detect a lesion effect during the immediate postoperative period. ${ }^{37}$ Reorganization of brain circuitry with chronic stimulation even in the absence of a histologic lesion may be an alternative expla- nation for seizure control maintained without ongoing stimulation.

Other studies of intermittent ANT DBS have reported significant reduction in frequency and propagation of generalized tonic-clonic seizures and complex partial seizures in four of five patients. ${ }^{35}$ Only one of the five patients exhibited a statistically significant reduction in total seizure frequency, however, and discontinuation of DBS resulted in an immediate increase in seizure frequency and intensity that improved when stimulation was resumed. This suggests that ANT DBS-induced inhibition is at least in part implicated in reducing seizure frequency. ${ }^{34,35,37}$ The beneficial response to closed-loop stimulation (discussed below) provides further support for a beneficial role of electrical stimulation.

A recent study of long-term follow-up noted that electrode implantation and stimulation of the ANT resulted in a $>50 \%$ seizure reduction in five of six patients. However, significant benefit in two patients was not seen until years 5 and 6 with the addition of further adjuvant AEDs. ${ }^{39}$ There is evidence for a long-term effect of ANT DBS on the propensity to develop seizures, ${ }^{39}$ and indeed, ongoing clinical benefit after cessation of DBS has been noted in tremor. ${ }^{44}$ Bitemporal mesial epilepsy, as opposed to extratemporal or poorly localized seizures, ${ }^{35,36,39}$ may be most responsive to ANT DBS, given a recent report of a $>75 \%$ reduction in seizure frequency. ${ }^{37}$ Seizure frequency decreased by $93 \%$ in one patient, the most dramatic response ever reported. A higher mean frequency $(157 \mathrm{~Hz})$ may in part explain such an impressive response.

\section{Complications and adverse effects}

ANT DBS appears to be a safe, well-tolerated treatment for refractory epilepsy, with all of the reported complications and stimulation-induced adverse effects being mild and transient. Reported adverse effects due to stimulation are paranoid ideation, nystagmus, auditory hallucinations, anorexia, and lethargy. ${ }^{39}$ Complications due to the surgical procedure or hardware include a small right frontal hemorrhage without any permanent neurologic deficit, scalp erosion necessitating removal of the implanted system, infection of the right anterior chest, and accidental turning off of the stimulator. ${ }^{34,36,45}$

\section{OTHER ANATOMIC TARGETS FOR DEEP BRAIN STIMULATION FOR EPILEPSY}

Other potential targets are worth discussing, to provide a more thorough review of the potential for DBS in epilepsy. Some open-label clinical trials have reported DBS of various targets to treat pharmacoresistant seizures, demonstrating marginal efficacy in stimulating the centromedian nucleus of the thalamus (CMT), caudate nucleus, cerebellum, and STN. 
TABLE 1. Clinical Outcomes After Anterior Nucleus Deep Brain Stimulation in Six Pilot Studies

\begin{tabular}{lcccc}
\hline \multicolumn{1}{c}{ Series } & $N$ & $\begin{array}{c}\text { Average Postoperative } \\
\text { Follow-up, mo }\end{array}$ & Stimulation Type & $\begin{array}{r}\text { Reduction in Monthly } \\
\text { Seizure Frequency, \% }\end{array}$ \\
\hline Osorio et al. $(2007)^{37}$ & 4 & 36 & Cycling & $76^{*}$ \\
Lee et al. (2006) & 3 & 13 & Cycling & 75 \\
Lim et al. $(2007)^{34}$ & 4 & 44 & Continuous & 51 \\
Hodaie et al. $(2002)^{36}$ & 5 & 15 & Cycling & $54^{\ddagger}$ \\
Kerrigan et al. $(2004)^{35}$ & 5 & 12 & Cycling & $14^{\S}$ \\
Andrade et al. $(2006)^{39}$ & 6 & 48 & Cycling $^{\ddagger}$ & $51^{\#}$ \\
\hline
\end{tabular}

$* p<0.01$.

'Intermittent stimulation was later used to prolong battery life, although the timescale was not reported.

${ }^{\ddagger} p<0.05$.

${ }^{\$}$ This study noted $>50 \%$ reduction in frequency of serious seizures (generalized tonic-clonic seizures and complex partial seizures).

${ }_{\text {Andrade et }}$ al. ${ }^{39}$ include results from patients included in the study by Hodaie et al. ${ }^{36}$

${ }^{9}$ Multiple changes in stimulation parameters, including continuous vs. cycling were performed in this study.

\#Four of the six patients had a significant decrease in the frequency of seizures/month $(P<0.05)$, but significance for overall mean reduction was not reported.

\section{Centromedian nucleus of the thalamus}

As first hypothesized by Penfield, ${ }^{46}$ the CMT is an integral part of an ascending subcortical system, arising from the brain stem and diencephalon and projecting diffusely to cerebral cortex, supporting a role for the CMT in the pathophysiology of generalized seizures. ${ }^{47}$ The beneficial effect of CMT DBS on seizure control may be due to desynchronization and hyperpolarization of the ascending reticular and cortical neurons. ${ }^{47}$

A recent open-label trial in 13 patients with LennoxGastaut syndrome reported overall seizure reduction of $80 \%$, with significant improvement in quality of life. ${ }^{48}$ Although CMT DBS may be effective in controlling frequency of generalized seizures, results in patients with complex partial seizures are unclear. ${ }^{49-52}$ In contrast to the results of these open-label studies, a trial of CMT DBS in seven patients using a double-blind, cross-over design, revealed no significant difference in seizure frequency, ${ }^{53}$ and a recent report indicated no short-term benefit in two patients, one of whom was subsequently implanted with electrodes in the ANT. ${ }^{39}$

\section{Subthalamic nucleus}

Extensive experience of STN DBS for the treatment of Parkinson's disease makes the STN an appealing target. ${ }^{8}$ The substantia nigra pars reticulata $(\mathrm{SNr})$ plays a role in seizure propagation through its GABAergic nigrotectal projections to the superior colliculus. ${ }^{54}$ Inhibition of GABAergic $\mathrm{SNr}$ neurons results in suppression of partial and generalized epileptic seizures in a rat model of epilepsy. ${ }^{55}$ The STN exerts excitatory control on the nigral system. Indeed, pharmacological or electrical inhibition of the STN leads to seizure suppression in rats. ${ }^{20}$

A case study of high-frequency bilateral STN DBS for a child with inoperable epilepsy due to focal centroparietal dysplasia observed an $81 \%$ improvement in seizure frequency at 30 months follow-up. ${ }^{56}$ An $80 \%$ improvement in two of five patients with partial-onset seizures treated with STN DBS for 16 months has been reported. ${ }^{57} \mathrm{An}$ other open-label study of STN DBS demonstrated significant improvement of seizures in 4 of 5 patients (mean reduction of seizure frequency $64.2 \%) .{ }^{58}$ Most recently, two cases with refractory partial-onset seizures were treated with bilateral STN DBS. ${ }^{59}$ The first patient had a $50 \%$ reduction in seizure frequency, but seizure-related injuries continued to occur. The second case noted seizure frequency to be reduced by one-third, but seizures were milder and caused fewer injuries, thus improving the quality of life of this patient. Larger, randomized, double-blind controlled trials are necessary to establish the potential of STN DBS as a treatment for epilepsy.

\section{Caudate nucleus}

The head of the caudate nucleus (HCN), thalamus, and neocortex are connected as a functional entity known as the caudate loop. ${ }^{60}$ Activation of the $\mathrm{HCN}$ is associated with hyperpolarization of cortical neurons, suggesting that suppression of seizure activity may be a result of stimulation-induced inhibition on the cortex. Indeed, low-frequency stimulation $(4-8 \mathrm{~Hz})$ of the ventral part of the HCN has been shown to suppress interictal activity, focal amygdalohippocampal discharges, and generalization of seizures. ${ }^{61,62}$ Controlled clinical studies are necessary to further evaluate the $\mathrm{HCN}$ as a target for DBS in epilepsy.

\section{Cerebellum}

Cerebellar stimulation (CS) likely activates inhibitory Purkinje cells, resulting in suppression of excitatory cerebellar output to the thalamus and decreased excitatory thalamocortical projections. This ultimately provides inhibition of cortical excitability. ${ }^{63}$

In the 1970s, Cooper et al. ${ }^{64,65}$ were the first to report on CS for epilepsy in an open-label trial, demonstrating that 18 of the 34 patients experienced a reduction in seizure frequency of $\geq 50 \%$. A later long-term study 
demonstrated that 23 of 32 patients $(85 \%)$ benefited overall from long-term CS. ${ }^{66}$ Controlled, double-blind trials, however, demonstrated improvement in only 2 of 14 patients (14\%). ${ }^{67,68} \mathrm{~A}$ recent double-blind trial of 5 patients demonstrated a significant reduction in generalized tonic-clonic seizures and tonic seizures at 2 years follow-up. ${ }^{69}$ Improvement in generalized tonic-clonic seizures occurred sooner, however, and was more significant than that for tonic seizures. Therefore, as suggested in animal studies, CS may act mainly at the supradiencephalic level. ${ }^{70}$ Larger clinical trials are necessary to further assess the role of CS in epilepsy treatment.

\section{Hippocampus}

Substantial evidence exists to suggest that temporal lobe seizures are both initiated from and propagated through the hippocampus, ${ }^{71,72}$ an integral component of the circuit of Papez. ${ }^{26,27,73}$ Subacute hippocampal stimulation (HS) using bilateral depth electrodes or unilateral electrode grids abolished complex partial and secondarily generalized tonic-clonic seizures and significantly decreased the number of interictal EEG spikes at the focus in 7 of 10 patients with intractable temporal lobe seizures. $^{74}$ A subsequent study of chronic HS by the same group in three patients demonstrated persistently blocked epileptogenesis with no negative effect on shortterm memory. ${ }^{75}$ Continuous high-frequency HS for about 2 weeks in 10 patients revealed a significant decrease in seizures and interictal paroxysmal activity. ${ }^{75}$

A small open series of three patients with complex partial seizures reported that DBS of the amygdalohippocampal region resulted in a $>50 \%$ reduction in seizure frequency at 5 months follow-up. ${ }^{76}$ More recently, these investigators reported that at a mean follow up of 14 months, bilateral amygdalohippocampal DBS in seven patients resulted in one patient who was free of complex partial seizures. ${ }^{77}$ Three other patients had more than a $50 \%$ reduction in seizure frequency, and two of the seven patients had a reduction of $25 \%$. Only one patient had no change in seizure frequency. Controlled studies with larger sample sizes are mandatory to identify a potential treatment population for HS and optimal stimulation parameters.

\section{OPEN- VERSUS CLOSED-LOOP SYSTEMS}

Closed-loop systems are so-called intelligent brain devices that produce bursts of stimulation in response to physiological changes and are capable of terminating epileptiform activity. ${ }^{11}$ These stimulators may provide comparable or even more effective seizure suppression than their blind, open-loop counterparts (described above), which stimulate continuously or intermittently. Furthermore, closed-loop devices are more efficient and should be better tolerated than an open-loop modality because the daily doses of stimulation are lower. ${ }^{78}$ Intelligent detection may be less toxic than either intermittent or continuous stimulation. ${ }^{11}$

Penfield and Jasper ${ }^{79}$ were the first to apply focal electrical stimulation to a human's brain and successfully terminated spontaneous seizures. A research group ${ }^{80}$ at the University of Pennsylvania, using EEG recordings in mesial temporal lobe epilepsy, identified a spontaneous progression of changes in brain activity that can lead to a full-blown convulsive seizure. These changes can last up to 7 hours prior to the seizure, and, if detected, can be successfully suppressed by an implanted stimulator. Mapping cortical networks involved in seizure generation and determining the most effective time of stimulation relative to seizure onset still require further investigation.

A trial of four patients with refractory seizures treated with responsive cortical stimulation noted suppression of clinical seizures and resolution of electrographic seizure activity. ${ }^{81}$ Recently, high-frequency stimulation was performed in eight patients, using a closed-loop system in which stimulation was delivered either to the epileptogenic cortex $(n=4)$ or the ANT $(n=4)$ after automated seizure detection. ${ }^{82}$ Three of the four cortical stimulation patients and two of the four ANT DBS patients responded with decreased seizure frequency.

A multi-institutional clinical trial of a cranially implanted responsive neurostimulator (RNS; NeuroPace, Mountain View, CA) is currently underway in patients with pharmacoresistant partial onset seizures. ${ }^{83}$ The RNS IPG continuously analyzes the patient's electrocortigram and triggers stimulation whenever the characteristics programmed by the clinician are indicative of seizures or epileptiform precursors. A feasibility study of this closedloop device has already described a decrease of $\sim 45 \%$ in seizure frequency in the majority of patients at 9 months follow-up. ${ }^{84}$

\section{CONCLUSION}

Many patients suffering from medically refractory epilepsy are not candidates for resective brain surgery. Some of these patients may benefit from DBS. There are many potential targets for DBS in epilepsy, but current evidence is limited to open-label pilot studies and very small double-blind clinical trials. Although a prospective double-blind randomized trial for ANT DBS is currently ongoing, ${ }^{25}$ it is difficult to make any definitive judgments about the efficacy of ANT DBS for seizure control. Further work is also necessary to identify a potential patient population for whom this technique would be indicated, which target is most efficacious, optimal stimulation parameters, and the ideal mode of stimulation. 


\section{REFERENCES}

1. Sillanpää M, Schmidt D. Natural history of treated childhood-onset epilepsy: prospective, long-term population-based study. Brain 2006;129:617-624.

2. Sander JW. Some aspects of prognosis in the epilepsies: a review. Epilepsia 1993;34:1007-1016.

3. Juul-Jensen P. Epidemiology of intractable epilepsy. In: Schmidt D, Morselli PL, editors. Intractable epilepsy: experimental and clinical aspects, 1st ed. New York: Raven Press, 1986:5-11.

4. A global survey on epilepsy surgery, 1980-1990: a report by the Commission on Neurosurgery of Epilepsy, the International League Against Epilepsy. Epilepsia 1997;38:249-255.

5. Kwan P, Brodie MJ. Early identification of refractory epilepsy. N Engl J Med 2000;342:314-319.

6. A randomized controlled trial of chronic vagus nerve stimulation for treatment of medically intractable seizures. The Vagus Nerve Stimulation Study Group. Neurology 1995;45:224-230.

7. Krack P, Batir A, Van Blercom N, et al. Five-year follow-up of bilateral stimulation of the subthalamic nucleus in advanced Parkinson's disease. N Engl J Med 2003;349:1925-1934.

8. Halpern C, Hurtig H, Jaggi J, Grossman M, Won M, Baltuch G. Deep brain stimulation in neurologic disorders. Parkinsonism Relat Disord 2007;13:1-16.

9. Schuurman PR, Bosch DA, Bossuyt PM, et al. A comparison of continuous thalamic stimulation and thalamotomy for suppression of severe tremor. N Engl J Med 2000;342:461-468.

10. Deogaonkar M, Boulis N, Machado A, Azmi H, Senatus P, Rezai A. Surgical complications in 800 consecutive DBS implants. Abstract. Presented at Annu Meet Am Assoc Neurol Surgeons (AANS), April 14-19, 2007, Washington, DC. Available at http:// www.aans.org [article ID 40777].

11. Litt B. Evaluating devices for treating epilepsy. Epilepsia 2003;44 Suppl 7:30-37.

12. Litt B, Baltuch G. Brain stimulation for epilepsy. Epilepsy Behav 2001;2:S61-S67.

13. Benabid AL, Pollak P, Louveau A, Henry S, de Rougemont J. Combined (thalamotomy and stimulation) stereotactic surgery of the VIM thalamic nucleus for bilateral Parkinson disease. Appl Neurophysiol 1987;50:344-346.

14. Lee KH, Roberts DW, Kim U. Effect of high-frequency stimulation of the subthalamic nucleus on subthalamic neurons: an intracellular study. Stereotact Funct Neurosurg 2003;80:32-36.

15. McIntyre CC, Savasta M, Kerkerian-Le Goff L, Vitek JL. Uncovering the mechanism(s) of action of deep brain stimulation: activation, inhibition, or both. Clin Neurophysiol 2004;115:12391248.

16. Monnier M, Kalberer M, Krupp P. Functional antagonism between diffuse reticular and intralaminary recruiting projections in the medial thalamus. Exp Neurol 1960;2:271-289.

17. Velasco M, Velasco F. Brain stem regulation of cortical and motor excitability: effects on experimental focal motor seizures. In: Sterman MB, Shouse MN, Passouant P, editors. Sleep and epilepsy. New York: Academic Press, 1982:53-61.

18. Lado FA, Velíšek L, Moshe SL. The effect of electrical stimulation of the subthalamic nucleus on seizures is frequency dependent. Epilepsia 2003;44:157-164.

19. Mullan S, Vailati G, Karasick J, Mailis M. Thalamic lesions for the control of epilepsy: a study of nine cases. Arch Neurol 1967;16: 277-285.

20. Vercueil L, Benazzouz A, Deransart C, et al. High-frequency stimulation of the subthalamic nucleus suppresses absence seizures in the rat: comparison with neurotoxic lesions. Epilepsy Res 1998; 31:39-46.

21. Shandra AA, Godlevsky LS. Antiepileptic effects of cerebellar nucleus dentatus electrical stimulation under different conditions of brain epileptisation. Indian J Exp Biol 1990;28:158-161.

22. Mirski MA, McKeon AC, Ferrendelli JA. Anterior thalamus and substantia nigra: two distinct structures mediating experimental generalized seizures. Brain Res 1986;397:377-380.

23. Bragin A, Wilson CL, Engel J Jr. Rate of interictal events and spontaneous seizures in epileptic rats after electrical stimulation of hippocampus and its afferents. Epilepsia 2002;43 Suppl 5:81-85.
24. Mutani R. Experimental evidence for the existence of an extrarhinencephalic control of the activity of the cobalt rhinencephalic epileptogenic focus. Part 1 . The role played by the caudate nucleus. Epilepsia 1969;10:337-350.

25. Fisher R; SANTE Study Group. SANTE (Stimulation of the Anterior Nucleus of Thalamus for Epilepsy) interim report. Abstract 4.122. Presented at: Annu Meet 60th Am Epilepsy Soc, 2006, San Diego, CA [Available at: http://www.aesnet.org].

26. Papez JW. A proposed mechanism of emotion. Arch Neurol Psychiatry 1937;38:725-743.

27. Oikawa H, Sasaki M, Tamakawa Y, Kamei A. The circuit of Papez in mesial temporal sclerosis: MRI. Neuroradiology 2001;43:205210.

28. Mirski MA, Ferrendelli JA. Selective metabolic activation of the mammillary bodies and their connections during ethosuximideinduced suppression of pentylenetetrazol seizures. Epilepsia 1986; 27:194-203.

29. Mirski MA, Ferrendelli JA. Interruption of the mammillothalamic tract prevents seizures in guinea pigs. Science 1984;226:72-74.

30. Mirski MA, Rossell LA, Terry JB, Fisher RS. Anticonvulsant effect of anterior thalamic high frequency electrical stimulation in the rat. Epilepsy Res 1997;28:89-100.

31. Dempsey EW, Morrison RS. The production of rhythmically recurrent cortical potentials after localized thalamic stimulation. Am J Physiol 1941;135:293-300.

32. Hamani C, Ewerton FI, Bonilha SM, Ballester G, Mello LE, Lozano AM. Bilateral anterior thalamic nucleus lesions and highfrequency stimulation are protective against pilocarpine-induced seizures and status epilepticus. Neurosurgery 2004;54:191-195; discussion 195-197.

33. Lado FA. Chronic bilateral stimulation of the anterior thalamus of kainate-treated rats increases seizure frequency. Epilepsia 2006; 47:27-32.

34. Lim SN, Lee ST, Tsai YT, et al. Electrical stimulation of the anterior nucleus of the thalamus for intractable epilepsy: a longterm follow-up study. Epilepsia 2007;48:342-347.

35. Kerrigan JF, Litt B, Fisher RS, et al. Electrical stimulation of the anterior nucleus of the thalamus for the treatment of intractable epilepsy. Epilepsia 2004;45:346-354.

36. Hodaie M, Wennberg RA, Dostrovsky JO, Lozano AM. Chronic anterior thalamus stimulation for intractable epilepsy. Epilepsia 2002;43:603-608.

37. Osorio I, Overman J, Giftakis J, Wilkinson SB. High frequency thalamic stimulation for inoperable mesial temporal epilepsy. Epilepsia 2007;48:1561-1571.

38. Samadani U, Baltuch GH. Anterior thalamic nucleus stimulation for epilepsy. Acta Neurochir Suppl 2007;97:343-346.

39. Andrade DM, Zumsteg D, Hamani C, et al. Long-term follow-up of patients with thalamic deep brain stimulation for epilepsy. Neurology 2006;66:1571-1573.

40. Schaltenbrand G, Hassler RG, Wahren W. Atlas for stereotaxy of the human brain, 2nd ed. Stuttgart: Thieme, 1977.

41. Zumsteg D, Lozano AM, Wennberg RA. Rhythmic cortical EEG synchronization with low frequency stimulation of the anterior and medial thalamus for epilepsy. Clin Neurophysiol 2006;117:22722278.

42. Upton AR, Amin I, Garnett S, Springman M, Nahmias C, Cooper IS. Evoked metabolic responses in the limbic-striate system produced by stimulation of anterior thalamic nucleus in man. Pacing Clin Electrophysiol 1987;10:217-225.

43. Tasker RR. Deep brain stimulation is preferable to thalamotomy for tremor suppression. Surg Neurol 1998;49:145-153; discussion $153-154$.

44. Lang AE, Lozano AM. Parkinson's disease. Second of two parts. N Engl J Med 1998;339:1130-1143.

45. Lee KJ, Jang KS, Shon YM. Chronic deep brain stimulation of subthalamic and anterior thalamic nuclei for controlling refractory partial epilepsy. Acta Neurochir Suppl 2006;99:87-91.

46. Penfield WB. The cerebral cortex in man. I. The cerebral cortex and consciousness (Harvey Lecture, 1936). Arch Neurol Psychiatry $1938 ; 40: 417$.

47. Velasco M, Velasco F, Velasco AL, Jiménez F, Brito F, Márquez I. Acute and chronic electrical stimulation of the centromedian 
thalamic nucleus: modulation of reticulo-cortical systems and predictor factors for generalized seizure control. Arch Med Res 2000;31:304-315.

48. Velasco AL, Velasco F, Jiménez F, et al. Neuromodulation of the centromedian thalamic nuclei in the treatment of generalized seizures and the improvement of the quality of life in patients with Lennox-Gastaut syndrome. Epilepsia 2006;47:1203-1212.

49. Velasco F, Velasco M, Ogarrio C, Fanghanel G. Electrical stimulation of the centromedian thalamic nucleus in the treatment of convulsive seizures: a preliminary report. Epilepsia 1987;28:421430.

50. Velasco M, Velasco F, Velasco AL, Velasco G, Jiménez F. Effect of chronic electrical stimulation of the centromedian thalamic nuclei on various intractable seizure patterns: II. Psychological performance and background EEG activity. Epilepsia 1993;34:10651074.

51. Velasco F, Velasco M, Velasco AL, Jiménez F, Márquez I, Rise M. Electrical stimulation of the centromedian thalamic nucleus in control of seizures: long-term studies. Epilepsia 1995;36:63-71.

52. Velasco F, Velasco M, Jiménez F, Velasco AL, Márquez I. Stimulation of the central median thalamic nucleus for epilepsy. Stereotact Funct Neurosurg 2001;77:228-232.

53. Fisher RS, Uematsu S, Krauss GL, et al. Placebo-controlled pilot study of centromedian thalamic stimulation in treatment of intractable seizures. Epilepsia 1992;33:841-851.

54. Gale K. Role of the substantia nigra in GABA-mediated anticonvulsant actions. Adv Neurol 1986;44:343-364.

55. Iadarola MJ, Gale K. Substantia nigra: site of anticonvulsant activity mediated by gamma-aminobutyric acid. Science 1982;218: 1237-1240.

56. Benabid AL, Koudsie A, Benazzouz A, et al. Deep brain stimulation of the corpus luysi (subthalamic nucleus) and other targets in Parkinson's disease: extension to new indications such as dystonia and epilepsy. J Neurol 2001;248 Suppl 3:III37-47.

57. Loddenkemper T, Pan A, Neme S, et al. Deep brain stimulation in epilepsy. J Clin Neurophysiol 2001;18:514-532.+

58. Chabardes S, Kahane P, Minotti L, Koudsie A, Hirsch E, Benabid AL. Deep brain stimulation in epilepsy with particular reference to the subthalamic nucleus. Epileptic Disord 2002;4 Suppl 3:S83S93.

59. Handforth A, DeSalles AA, Krahl SE. Deep brain stimulation of the subthalamic nucleus as adjunct treatment for refractory epilepsy. Epilepsia 2006;47:1239-1241.

60. Heuser G, Buchwald NA, Wyers EJ. The "caudatespindle". II. Facilitatory and inhibitory caudate-cortical pathways. Electroencephalogr Clin Neurophysiol 1961;13:519-524.

61. Chkhenkeli SA, Sramka M, Lortkipanidze GS, et al. Electrophysiological effects and clinical results of direct brain stimulation for intractable epilepsy. Clin Neurol Neurosurg 2004;106:318-329.

62. Chkhenkeli SA, Chkhenkeli IS. Effects of therapeutic stimulation of nucleus caudatus on epileptic electrical activity of brain in patients with intractable epilepsy. Stereotact Funct Neurosurg 1997;69:221-224.

63. Molnar GF, Sailer A, Gunraj CA, Lang AE, Lozano AM, Chen R. Thalamic deep brain stimulation activates the cerebellothalamocortical pathway. Neurology 2004;63:907-909.

64. Cooper IS, Amin I, Gilman S. The effect of chronic cerebellar stimulation upon epilepsy in man. Trans Am Neurol Assoc 1973; 98:192-196.

65. Cooper IS, Amin I, Riklan M, Waltz JM, Poon TP. Chronic cer- ebellar stimulation in epilepsy. Clinical and anatomical studies. Arch Neurol 1976;33:559-570.

66. Davis R, Emmonds SE. Cerebellar stimulation for seizure control: 17-year study. Stereotact Funct Neurosurg 1992;58:200-208.

67. Van Buren JM, Wood JH, Oakley J, Hambrecht F. Preliminary evaluation of cerebellar stimulation by double-blind stimulation and biological criteria in the treatment of epilepsy. J Neurosurg 1978;48:407-416.

68. Wright GD, McLellan DL, Brice JG. A double-blind trial of chronic cerebellar stimulation in twelve patients with severe epilepsy. J Neurol Neurosurg Psychiatry 1984;47:769-774.

69. Velasco F, Carrillo-Ruiz JD, Brito F, et al. Double-blind, randomized controlled pilot study of bilateral cerebellar stimulation for treatment of intractable motor seizures. Epilepsia 2005;46:1071-1081.

70. Laxer KD, Robertson LT, Julien RM, Dow RS. Phenytoin: relationship between cerebellar function and epileptic discharges. Adv Neurol 1980;27:415-427.

71. Swanson TH. The pathophysiology of human mesial temporal lobe epilepsy. J Clin Neurophysiol 1995;12:2-22.

72. Sperling MR, O'Connor MJ, Saykin AJ, et al. A noninvasive protocol for anterior temporal lobectomy. Neurology 1992;42:416-422.

73. Carpenter MB. Core text of neuroanatomy, 4th ed. Baltimore: Williams \& Wilkins, 1991.

74. Velasco AL, Velasco M, Velasco F, et al. Subacute and chronic electrical stimulation of the hippocampus on intractable temporal lobe seizures: preliminary report. Arch Med Res 2000;31:316328.

75. Velasco F, Velasco M, Velasco AL, Menez D, Rocha L. Electrical stimulation for epilepsy: stimulation of hippocampal foci. Stereotact Funct Neurosurg 2001;77:223-227.

76. Vonck K, Boon P, Achten E, De Reuck J, Caemaert J. Long-term amygdalohippocampal stimulation for refractory temporal lobe epilepsy. Ann Neurol 2002;52:556-565.

77. Vonck K, Boon P, Claeys P, Dedeurwaerdere S, Achten R, Van Roost D. Long-term deep brain stimulation for refractory temporal lobe epilepsy. Epilepsia 2005;46 Suppl 5:98-99.

78. Osorio I, Frei MG, Manly BF, Sunderam S, Bhavaraju NC, Wilkinson SB. An introduction to contingent (closed-loop) brain electrical stimulation for seizure blockage, to ultra-short-term clinical trials, and to multidimensional statistical analysis of therapeutic efficacy. J Clin Neurophysiol 2001;18:533-544.

79. Penfield W, Jasper H. Epilepsy and the functional anatomy of the human brain. Boston:Little, Brown, 1954.

80. Litt B, Esteller R, Echauz J, et al. Epileptic seizures may begin hours in advance of clinical onset: a report of five patients. Neuron 2001;30:51-64.

81. Kossoff EH, Ritzl EK, Politsky JM, et al. Effect of an external responsive neurostimulator on seizures and electrographic discharges during subdural electrode monitoring. Epilepsia 2004;45: $1560-1567$.

82. Osorio I, Frei MG, Sunderam S, et al. Automated seizure abatement in humans using electrical stimulation. Ann Neurol 2005;57: $258-268$.

83. Sun FT, Morell MJ, Wharen RE. Responsive cortical stimulation for the treatment of epilepsy. Neurotherapeutics 2008;5:000-000 (this issue).

84. Fountas KN, Smith JR, Murro AM, Politsky J, Park YD, Jenkins PD. Implantation of a closed-loop stimulation in the management of medically refractory focal epilepsy: a technical note. Stereotact Funct Neurosurg 2005;83:153-158. 